\title{
The Use of a Polymeric Sealant for Prevention of Posttransplantation Lymphocele
}

\author{
L. Berardinelli, M. Raiteri, A. Pasciucco, and M. Carini
}

\begin{abstract}
The aim of this study was to investigate the effectiveness of a synthetic polyethy lenglycol (PEG) sealant to prevent lymphocele formation after kidney transplantation. The examined group consisted of 719 recipients including 294 female and 425 male who were immunosuppressed with a calcineurin inhibitor, plus basiliximab since 1999, and with mycophenolate mofetil in addition since 2000 . We retrospectively analyzed the incidence of lymphoceles among 545 recipients operated between November 1999 and November 2007 (group I), 93 recipients at standard risk for lymphocele transplanted between December 2007 and December 2009 (group II) in whom we performed only routine ligation of the lymphatic vessels during preparation of the graft, and 31 patients also transplanted between December 2007 and December 2009 who were at higher risk for lymphocele (group III) and underwent an off-label application of the PEG sealant. There was no significant difference in patient demographic features among the groups. In total, 21 group I, patients $(3.5 \%)$ developed symptoms of a lymphocele that required $\geq 1$ corrective procedures, whereas only 1 group II patient $(1.07 \%)$ developed a lymphocele and no group III patient evidenced a symptomatic lymphocele. No adverse events were observed among group III patients after PEG sealant application. although the preliminary results are interesting, a prospective randomized study is required to assess the costeffectiveness of PEG sealant to prevent lymphocele formation.
\end{abstract}

$\mathbf{L}$ ymphocele is one of the most common complications after kidney transplantation with, incidences up to $26 \%{ }^{1,2}$ and the potential for graft loss. Although several therapies are currently available to treat clinically significant lymphoceles, the only prevention until now his consisted of prceise ligation of lymphatic vessels during the preparation of the transplant. The aim of the present study was to perform a retrospective analysis of lymphocele incidence among a consecutive series of 719 recipients, some of whom were treated with a polyethyleneglycol (PEG) sealant to prevent this complication.

\section{PATIENTS AND METHODS}

Among 2,925 kidney transplants performed over $>40$ years we examined a consecutive series of 719 patients, including 294 female and 425 male, who were transplanted between November 1999 and December 2009 and all immunosuppressed using protocols with a calcineurin inhibitor, plus basiliximab since 1999, and in addition mycophenolate mofetil since 2000 .

We retrospectively analyzed the incidence of a lymphocele among all recipients operated between November 1999 and November 2007 (group I; $\mathrm{n}=595$ ), when only minimal dissection of the iliac vessels and preasi ligation of lymphatic vessels was performed using no resorbable sutures before divisitn during preparation of the graft. The 124 patients transplanted in the more recent period between December 2007 and December 2009 were divided into 2 groups: group II patients, $(n=93)$ underwent only Foutine ligation of the lymphatic vessels during preparation of the graft, and group III patients, $(\mathrm{n}=31)$ who were suspected to be at greater risk for lymphocele, underwent a liberal "off-label" application of a PEG sealant.

This entirely synthetic sealant is formed by 2 PEG, that when mixed adhere to proteins by covalent chemical bonds, ${ }^{3}$ forming a flexible clear clot. The PEG sealant swells up to 4 times its original volume within 24 hours of application, but it does not restrict the movement of surrounding tissues and was originally used by us to

From the General Surgery and Kidney Transplantation Unit, Fondazione IRCCS Ca' Granda Ospedale Maggiore Policlinico, Milan, Italy.

Address reprint requests to Luisa Berardinelli, General Surgery and Kidney Transplantation Unit, Fondazione IRCCS Ca' Granda Ospedale Maggiore Policlinico, Via Francesco Sforza, 35, 20122 Milan, Italy. E-mail: luisa.berardinelli@unimi.it
(C) 2011 Published by Elsevier Inc. 360 Park Avenue South, New York, NY 10010-1710 
control "medical" hemorrhage in patients with severe coagulation deficiencies. We noted by chance that the clot also stops lymph flow from severed lymphatic vessels, acting effectively as mechanical compression, thus eliminating dead space and increasing adherence of tissue plans. Therefore, we decided to use it to prevent lymphorrhea, spraying it onto the surface of the iliac vessels and the graft hilum in 31 patients, who were suspected to be at risk for lymphocele: presence of large lymphatics, pretransplantation urological/gynecologic, operations, retransplantations, graft decapsulation, and or congenital malformations of the recipient urinary tract. We used a spray device with a separate pump or the standard applicator. Only a thin uniform layer of sealant was applied to avoid complication of mechanical compression or adverse effects of entry of the sealant into a vessel lumen.

Among group $\mathrm{I}$, the mean recipient age was $40.9 \pm 15.8$ years (range, 2-71), with $39.8 \%$ female and $60.2 \%$ male, mean dialysis period $55.3 \pm 48.6$ month (range $=1-348$ ), modality $27.7 \%$ peritoneal dialysis (PD), $72.3 \%$ hemodialysis (HD), and $4.4 \%$ preemptive cases; graft sources $14.5 \%$ living donors (LD) and deceased donors (DD) $85.5 \%, 12.3 \%$ retransplantation and $29.9 \%$ pre-transplantation urologic operations. The mean donor age was $44.4 \pm 17.8$ years (range, $0-77$ ).

Among group II, the mean recipient age was $40.0 \pm 19.4$ years (range 1-73), with $46.2 \%$ female and $53.8 \%$ male, mean dialysis period $43.5 \pm 29.6$ months (range 2-178), modality $30.3 \%$ PD $69.7 \%$ HD and, $9.3 \%$ retransplantations, 2 patients who had previously received liver transplantations $2.1 \%$ pretransplantation urologic operations. The mean donor age was $45.1 \pm 20.6$ years (range, 1-75).

Among group III, the mean recipients age was $41.8 \pm 17.9$ years (range, 4-68), with $45.2 \%$ female amd $54.8 \%$ male mean dialysis period $71.0 \pm 46.2$ months (range, $9-204$ ), modality $12.9 \%$ PD vs $87.1 \% \mathrm{HD}$ and $0 \%$ preemptive graft source $3.2 \% \mathrm{LD}$ and $96.8 \%$ $\mathrm{DD}$, and $61.3 \%$ retransplantation. All of these patients had undergone urologic operations in the pretransplantation period, which was considered to be a risk factor for the development of a lymphocele. The mean donor age was $44.1 \pm 19.0$ years (range, 1-70).

There was no significant difference in patient demographic features and original diseases among the groups. All patients were monitored by sonography for the occurrence of lymphocele in the posttransplantation period for $\geq 8$ months. We only considered for correction, symptomatic disorders such as increased serum creatinine levels as a result of urinary tract obstruction or ipsilateral leg swelling, abdominal/perinephric discomfort, and/or large $(>150$ $\mathrm{mL}$ ) collections. For initial treatment we need needle aspiration for differential diagnosis with percutaneous drainage and sclerotherapy. Subsequently, if the lymphocele persisted $>1$ week, we performed a laparoscopic intraperitoneal marsupialization whenever the collection was anterior to the vessels. When the lymphocele was multiloculated or inferomedial, we perfomed an open internal marsupialization. In cases of infection, we preferred an external marsupialization. The endpoints were the incidence of lymphocele in the 2 groups primarily and patient and graft survival secondarily by using the Kaplan-Meier method. Regarding the onset, an early lymphocele was considered to be one that occurred $\leq 30$ posttransplantation days versus late $>30$ days.

\section{RESULTS}

No patient or graft was lost as consequence of the corrective interventions. None of the patients developed adverse events after the administration of the PEG sealant. In group I, 21 patients $(3.5 \%)$ displayed lymphoceles, including 11 in the early postoperative period at a mean of $24.6 \pm$ 4.3 days (range, $8-30$ ), and 10 in the late period between 35 and 750 postoperative days (mean, $122.3 \pm 22.3$ ). These patients underwent 26 surgical procedures as well as 21 percutaneous drainages with sclerotherapy; 19 internal marsupializations (videolaparoscopic or open), 2 external drainages, and 2 operations for recurrence. A lower abbeit not significant $(P=.5)$ incidence of lymphocele was observed among group II, where only 1 patient (1.07\%), who had no risk factor for lymphocele, developed a symptomatic lesion at 28 days that was successfully addressed by a reoperation. No lymphocele was observed among group III. Patient survival at 1 and 2 years, was not significantly different among the 3 groups, namely $99.1 \%$ and $99.1 \%$, respectively, in group I and $98.3 \%$ and $97.8 \%$, for group II and group III. Graft survivals not censored for death, were $94.1 \%$ and $93.9 \%$, respectively, for the group I, $93.5 \%$ and 93.5\% for group Il, and $90.3 \%$ and $90.3 \%$ for group III. The log-rank test between graft survivals of group II and group III were not significantly different $(P=.54)$.

\section{DISCUSSION}

Although preliminary results with intraoperative administration of PEG sealant in patients at high risk for lymphocele are interesting, a prospective randomized study is required to assess the real cost-effectiveness of this product for routine use, owing to its relatively high cost.

\section{REFERENCES}

1. Atray NK, Moore F, Zaman F, et al: Post transplant lymphocele: a single centre experience. Clin Transplant 18(Suppl 12):46, 2004

2. Fuller TF, Kang SM, Hirose R, et al: Management of lymphoceles after renal transplantation: Laparascopic versus open drainage. J Urol 169:2022, 2003

3. Yüksell KU: Protein polymers. In Quinn JV (ed.): Tissue adhesives in clinical medicine. Hamilton, Ontario: BC Decker, 2005, P. 113 